\title{
Research Article \\ Robustness of Exponential Dissipation with respect to Small Time Delay
}

\author{
Xunwu Yin \\ School of Science, Tianjin Polytechnic University, Tianjin 300387, China \\ Correspondence should be addressed to Xunwu Yin; yinxunwu@hotmail.com
}

Received 22 March 2013; Revised 20 May 2013; Accepted 24 May 2013

Academic Editor: Yuri V. Rogovchenko

Copyright (C) 2013 Xunwu Yin. This is an open access article distributed under the Creative Commons Attribution License, which permits unrestricted use, distribution, and reproduction in any medium, provided the original work is properly cited.

We investigate robustness of exponential dissipation for the following general nonlinear evolutionary equation with small time delay: $\partial_{t} u+A u=f(u(t), u(t-\tau))$. We firstly obtain a converse Lyapunov theorem. With the help of it, we establish an important result on robustness of exponential dissipation to small time delay assuming that the nonlinearity is globally Lipschitz.

\section{Introduction}

As is well known, time delays are usually encountered in practical control systems. The stability analysis has received attentions over the last several decades. Mathematically, it is also very important to understand the sensitivity of the dynamical behavior of the system to the introduction of small time delays. For linear systems, we well understand this problem, including both finite dimensional and infinite dimensional situations, see [1-5]. However, for nonlinear systems, the problem is much more difficult, but there are some very nice results in [6-10].

This paper is devoted to the following general nonlinear evolutionary equation with small delay:

$$
\partial_{t} u+A u=f(u(t), u(t-\tau)),
$$

where the nonlinearity $f: X^{\alpha} \times X^{\alpha} \rightarrow X$ is assumed to be globally Lipschitz. Here, $A$ is a sectorial operator on a Banach space $X . X^{\alpha}$ is a fractional power space. Here, we investigate the effects of small time delay on the exponential dissipation of the corresponding evolutionary equation without delay:

$$
\partial_{t} u+A u=F(u)
$$

where $F(u)=f(u, u)$.

In [11], Lyapunov introduced his famous sufficient conditions for asymptotic stability of the following nonautonomous dynamical system:

$$
x^{\prime}(t)=f(t, x(t)), \quad x(t) \in R^{n} .
$$

There, we can also find the first contribution to the converse question, known as converse Lyapunov theorems. In recent years, the answers have proved instrumental in establishing robustness of various stability notions and have served as the starting point for many nonlinear control systems design concepts.

In 2005, Li and Kloeden [8] presented a converse Lyapunov theorem for exponential dissipation of the following general nonlinear differential equations with multiple small time delays:

$$
x^{\prime}(t)=f\left(x\left(t-\tau_{1}\right), x\left(t-\tau_{2}\right), \ldots, x\left(t-\tau_{n}\right)\right),
$$

where $f$ is assumed to be globally Lipschitz. They also prove that exponential dissipation remains under small time delays. This result can be seen as a generalization of some classical ones on global exponential asymptotic stability (e.g., [12]) and was used by the authors to study robustness of exponential dissipation with respect to small time delays.

Recently, Guo and Li [13] gave a nonautonomous analog of the result. They not only present a converse Lyapunov theorem but also prove robustness of the uniform exponential dissipation with respect to unbounded external perturbations.

In the dynamical theory, a basic problem concerns the robustness of global attractors under perturbations [14]. It is known that if a nonlinear system with a global attractor $\mathscr{A}$ is perturbed, then the perturbed one also has an attractor $\mathscr{A}^{\prime}$ near $\mathscr{A}$, provided that the perturbation is sufficiently small $[7,15]$. However, in general, we only know that $\mathscr{A}^{\prime}$ is a local 
attractor. Whether the global feature can be preserved is still an open problem. To our great joy, a dissipative system usually implies the existence of the global attractor. So, if one wants to settle the above problem, he only needs to examine the robustness of dissipation under perturbations. In this present work, we will investigate the infinite dimensional situations which are more difficult than the finite ones. With the nonlinearity being globally Lipschitz, we obtain a converse Lyapunov theorem and prove that exponential dissipation has nice robustness properties under small time delay.

\section{Preliminaries}

In this paper, we study the following delayed initial value problem:

$$
\begin{gathered}
u_{t}+A u=f(u(t), u(t-\tau)), \quad t>0, \\
\left.u\right|_{[-\tau, 0]}=u_{0}(t)
\end{gathered}
$$

For simplicity, we use $\|\cdot\|$ and $\|\cdot\|_{\alpha}$ to denote the norm on $X$ and $X^{\alpha}$, respectively. We write $\mathscr{C}=C\left([-\tau, 0], X^{\alpha}\right)$ with the norm $|\|\cdot\||_{\alpha}$ defined by

$$
\|\| u_{0}\left\|_{\alpha}=\max _{t \in[-\tau, 0]}\right\| u_{0}(t) \|_{\alpha}, \quad \forall u_{0} \in \mathscr{C} .
$$

Next, we will recall some basic definitions and facts.

The upper right Dini derivative of a function $y \in C((\alpha$, $\left.\beta), X^{\alpha}\right)$ is defined as

$$
\frac{d^{+}}{d t} y(t):=\limsup _{h \rightarrow 0+} \frac{y(t+h)-y(t)}{h}
$$

Let $x \in X^{\alpha}$ and $\mathcal{N}$ be an open neighborhood of $x$. For $V \in$ $C\left(\mathcal{N}, R^{1}\right)$ and $v \in X^{\alpha}$, we define

$$
D_{v}^{+} V(x):=\limsup _{h \rightarrow 0+} \frac{V(x+h v)-V(x)}{h} .
$$

We will denote by $u(t, x)$ the solution of (2), where $u(0, x)=x$.

Definition 1. The system (2) is said to be exponentially dissipative, if there exist positive numbers $B, \lambda$, and $\rho$ such that

$$
\|u(t, x)\|_{\alpha} \leq B e^{-\lambda t}\|x\|_{\alpha}+\rho, \quad \forall t \geq 0, x \in X^{\alpha} .
$$

Lemma 2. Let $\mathcal{N}$ be an open subset of $X^{\alpha}$. Assume that the function $V: \mathscr{N} \rightarrow R^{+}$is Lipschitz; that is, there exists a $L_{V}>0$ such that

$$
|V(x)-V(y)| \leq L_{V}\|x-y\|_{\alpha}, \quad \forall x, y \in X^{\alpha} .
$$

Let $u(t)$ be a solution of (2). Then,

$$
\frac{d^{+}}{d t} V(u(t))=D_{g(u)}^{+} V(u(t)), \quad \text { where } g(u)=F(u)-A u \text {. }
$$

Proof. The detailed proof is contained in $[12,16]$. Here, we give a simple proof for the reader's convenience. Making use of Taylor formula, we observe that

$$
\begin{aligned}
V(u(t+h))-V(u(t)) \\
=V(u(t)+g(u) h+o(h))-V(u(t)) \\
=V(u(t)+g(u) h+o(h))-V(u(t)+g(u) h) \\
\quad+V(u(t)+g(u) h)-V(u(t)) .
\end{aligned}
$$

Since $V(x)$ is Lipschitz, one easily sees that

$$
V(u(t)+g(u) h+o(h))-V(u(t)+g(u) h)=o(h) .
$$

Therefore, by definition (7), we immediately deduce that

$$
\begin{aligned}
& \frac{d^{+}}{d t} V(u(t)) \\
& \quad=\limsup _{h \rightarrow 0+} \frac{V(u(t+h))-V(u(t))}{h} \\
& \quad=\limsup _{h \rightarrow 0+} \frac{V(u(t)+g(u) h)-V(u(t))}{h} \\
& =D_{g(u)}^{+} V(u(t)) .
\end{aligned}
$$

The proof is finished.

At last, we come to the main theorem on analytic semigroup which is extremely important in the study of the dynamics of nonlinear evolutionary equations [17].

Theorem 3 (fundamental theorem on sectorial operators). Let $A$ be a positive, sectorial operator on a Banach space $X$ and let $e^{-A t}$ be the analytic semigroup generated by $-A$. Then, the following statements hold.

(1) For any $\alpha \geq 0$, there is a constant $C_{\alpha}>0$ such that for all $t>0$

$$
\left\|A^{\alpha} e^{-A t}\right\|_{L(X)} \leq C_{\alpha} t^{-\alpha} e^{-a t} \quad(a>0) .
$$

(2) For $0<\alpha \leq 1$, there is a constant $C_{\alpha}>0$ such that for $t \geq 0$ and $x \in D\left(A^{\alpha}\right)$

$$
\left\|e^{-A t} x-x\right\| \leq C_{\alpha} t^{\alpha}\left\|A^{\alpha} x\right\| .
$$

(3) For every $\alpha \geq 0$, there is a constant $C_{\alpha}>0$ such that for all $t>0$ and $x \in X$

$$
\left\|\left(e^{-A(t+h)}-e^{-A t}\right) x\right\|_{\alpha} \leq C_{\alpha}|h| t^{-(1+\alpha)}\|x\| .
$$

\section{Main Results}

In this section, we will prove our two main results: one is converse Lyapunov theorem, and the other is robustness of exponential dissipation to small time delay. 
Theorem 4 (converse Lyapunov theorem). Suppose that $F$ : $X^{\alpha} \rightarrow X$ in (2) is globally Lipschitz with Lipschitz constant L. Suppose that the system without delay (2) is exponentially dissipative. Then, there exists a function $V: X^{\alpha} \rightarrow R^{+}$satisfying

$$
\begin{gathered}
\|x\|_{\alpha}^{2}-a \leq V(x) \leq b\|x\|_{\alpha}^{2}+c, \\
D_{g(x)}^{+} V(x) \leq-d\|x\|_{\alpha}^{2}+\sigma, \\
|V(x)-V(y)| \leq L_{V}\left(\|x\|_{\alpha}+\|y\|_{\alpha}+1\right)\|x-y\|_{\alpha}
\end{gathered}
$$

for all $x, y \in X^{\alpha}$, where $g(x)=F(x)-A x, a, b, c, d, \sigma$, and $L_{V}$ are appropriate positive constants.

Proof. Since the system (2) is exponentially dissipative, there exist positive constants $B, \lambda$, and $\rho$ such that

$$
\|u(t, x)\|_{\alpha} \leq B e^{-\lambda t}\|x\|_{\alpha}+\rho, \quad \forall t \geq 0, x \in X^{\alpha} .
$$

Let $T=\ln (2 B) / \lambda$, and define $V_{1}$ as follows:

$$
V_{1}(x):=\int_{0}^{T}\|u(s, x)\|_{\alpha}^{2} d s, \quad x \in X^{\alpha} .
$$

By (21) and the elementary inequality, it is easy to check that

$$
\begin{aligned}
0 \leq V_{1}(x) & \leq \int_{0}^{T}\left(B e^{-\lambda s}\|x\|_{\alpha}+\rho\right)^{2} d s \\
& \leq 2 \int_{0}^{T}\left(B^{2} e^{-2 \lambda s}\|x\|_{\alpha}^{2}+\rho^{2}\right) d s \\
& \leq \frac{B^{2}}{\lambda}\|x\|_{\alpha}^{2}+2 T \rho^{2} .
\end{aligned}
$$

So, $V_{1}(x)$ satisfies the right inequality of (18).

Next, by the Lipschitz continuity of $F$, it is easy to verify that there exists a constant $C(T)>0$ such that

$$
\begin{array}{r}
\|u(t, x)-u(t, y)\|_{\alpha} \leq C(T)\|x-y\|_{\alpha} \\
\forall x, y \in X^{\alpha}, t \in[0, T] .
\end{array}
$$

Considering (21) and (23), for any $x, y \in X^{\alpha}$, we have

$$
\begin{aligned}
& \left|V_{1}(x)-V_{1}(y)\right| \\
& \quad=\left|\int_{0}^{T}\left(\|u(s, x)\|_{\alpha}^{2}-\|u(s, y)\|_{\alpha}^{2}\right) d s\right| \\
& \quad \leq C(T)\|x-y\|_{\alpha} \int_{0}^{T}\left(\|u(s, x)\|_{\alpha}+\|u(s, y)\|_{\alpha}\right) d s \\
& \quad \leq C(T)\|x-y\|_{\alpha} \int_{0}^{T}\left[B e^{-\lambda s}\left(\|x\|_{\alpha}+\|y\|_{\alpha}\right)+2 \rho\right] d s \\
& \quad \leq C(T)\|x-y\|_{\alpha}\left[\frac{B}{\lambda}\left(\|x\|_{\alpha}+\|y\|_{\alpha}\right)+2 \rho T\right] d s \\
& \quad \leq L_{1}\left(\|x\|_{\alpha}+\|y\|_{\alpha}+1\right)\|x-y\|_{\alpha} .
\end{aligned}
$$

So, $V_{1}(x)$ satisfies $(20)$.
Since

$$
\begin{aligned}
V_{1}(u(t, x)) & =\int_{0}^{T}\|u(s, u(t, x))\|_{\alpha}^{2} d s \\
& =\int_{0}^{T}\|u(t+s, x)\|_{\alpha}^{2} d s \\
& =\int_{t}^{t+T}\|u(s, x)\|_{\alpha}^{2} d s,
\end{aligned}
$$

by the choice of $T$ and (21), we have that

$$
\begin{aligned}
& \frac{d}{d t} V_{1}(u(t, x)) \\
& \quad=\|u(t+T, x)\|_{\alpha}^{2}-\|u(t, x)\|_{\alpha}^{2} \\
& \quad=\|u(T, u(t, x))\|_{\alpha}^{2}-\|u(t, x)\|_{\alpha}^{2} \\
& \quad \leq-\|u(t, x)\|_{\alpha}^{2}+\left(B e^{-\lambda T}\|u(t, x)\|_{\alpha}+\rho\right)^{2} \\
& \quad \leq-\|u(t, x)\|_{\alpha}^{2}+2 B^{2} e^{-2 \lambda T}\|u(t, x)\|_{\alpha}^{2}+2 \rho^{2} \\
& \quad \leq-\frac{1}{2}\|u(t, x)\|_{\alpha}^{2}+2 \rho^{2} .
\end{aligned}
$$

Consequently, by Lemma 2,

$$
D_{g(u(t, x))}^{+} V_{1}(u(t, x))=\frac{d^{+}}{d t} V_{1}(u(t, x))=\frac{d}{d t} V_{1}(u(t, x)) .
$$

In particular, setting $t=0$, one obtains that

$$
D_{g(x)}^{+} V_{1}(x)=\left.\frac{d}{d t} V_{1}(u(t, x))\right|_{t=0} \leq-\frac{1}{2}\|x\|_{\alpha}^{2}+2 \rho^{2},
$$

which indicates that $V_{1}$ satisfies (19).

Now, let us define another Lyapunov function $V_{2}$. We firstly take a nonnegative function $\gamma(s)$ as

$$
\gamma(s)=\max \left\{s^{2}-\rho_{0}^{2}, 0\right\}, \quad s \geq 0,
$$

where $\rho_{0}=(2 B+1) \rho$. It is easy to check that $\gamma(s)$ satisfies

$$
|\gamma(s)-\gamma(r)| \leq(s+r)|s-r|, \quad \forall s, r \geq 0 .
$$

Now, we let

$$
V_{2}(x)=\sup _{s \geq 0} \gamma\left(\|u(s, x)\|_{\alpha}\right), \quad \forall x \in X^{\alpha} .
$$

We firstly verify the following fact:

$$
V_{2}(x)=\sup _{0 \leq s \leq T} \gamma\left(\|u(s, x)\|_{\alpha}\right), \quad \forall x \in X^{\alpha} .
$$

Indeed, if $\|x\|_{\alpha} \leq 2 \rho$, then by (21)

$$
\|u(t, x)\|_{\alpha} \leq B e^{-\lambda t}\|x\|_{\alpha}+\rho \leq 2 \rho B+\rho=\rho_{0} .
$$


According to the definition of $\gamma(s)$, we know that $\gamma\left(\|u(s, x)\|_{\alpha}\right)=0$. Therefore, in case of $\|x\|_{\alpha} \leq 2 \rho$, one trivially has

$$
V_{2}(x)=0=\sup _{0 \leq s \leq T} \gamma\left(\|u(s, x)\|_{\alpha}\right) .
$$

If $\|x\|_{\alpha} \geq 2 \rho$, then by the choice of $T$ we find that

$$
\|u(s, x)\|_{\alpha} \leq B e^{-\lambda T}\|x\|_{\alpha}+\rho=\frac{\|x\|_{\alpha}}{2}+\rho<\|x\|_{\alpha}, \quad \forall s \geq T .
$$

Since $u(0, x)=x$ and $\gamma(s)$ is nondecreasing in $s$, one can deduce the correctness of (33).

Next, we will check that $V_{2}$ also satisfies (20). By (33), (31), (24), and (21)

$$
\begin{aligned}
V_{2}(x) & \sup _{0 \leq s \leq T} \gamma\left(\|u(s, x)\|_{\alpha}\right) \\
= & \sup _{0 \leq s \leq T}\left[\left(\gamma\left(\|u(s, x)\|_{\alpha}\right)-\gamma\left(\|u(s, y)\|_{\alpha}\right)\right)+\gamma\left(\|u(s, y)\|_{\alpha}\right]\right. \\
\leq & \sup _{0 \leq s \leq T}\left[\gamma\left(\|u(s, x)\|_{\alpha}\right)-\gamma\left(\|u(s, y)\|_{\alpha}\right)\right]+V_{2}(y) \\
\leq & \sup _{0 \leq s \leq T}\left(\|u(s, x)\|_{\alpha}+\|u(s, y)\|_{\alpha}\right) \\
& \times\left|\|u(s, x)\|_{\alpha}-\|u(s, y)\|_{\alpha}\right|+V_{2}(y) \\
\leq & C(T)\|x-y\|_{\alpha} \\
& \times \sup _{0 \leq s \leq T}\left[B e^{-\lambda s}\left(\|x\|_{\alpha}+\|y\|_{\alpha}\right)+2 \rho\right]+V_{2}(y) \\
\leq & C(T)\left[B\left(\|x\|_{\alpha}+\|y\|_{\alpha}\right)+2 \rho\right]\|x-y\|_{\alpha}+V_{2}(y) \\
\leq & L_{2}\left(\|x\|_{\alpha}+\|y\|_{\alpha}+1\right)\|x-y\|_{\alpha}+V_{2}(y) .
\end{aligned}
$$

Next, we will check that for arbitrary $x \in X^{\alpha}, V_{2}(x)$ is bounded by

$$
\|x\|_{\alpha}^{2}-\rho_{0}^{2} \leq V_{2}(x) \leq 2 B^{2}\|x\|_{\alpha}^{2}+2 \rho^{2} .
$$

Firstly, according to the definition of $\gamma(s)$, it is obvious to see that

$$
s^{2}-\rho_{0}^{2} \leq \gamma(s) \leq s^{2}, \quad \forall s \geq 0 .
$$

So, it follows that

$$
\begin{aligned}
V_{2}(x) & =\sup _{s \geq 0} \gamma\left(\|u(t, x)\|_{\alpha}\right) \geq \gamma\left(\|u(0, x)\|_{\alpha}\right) \\
& =\gamma\left(\|x\|_{\alpha}\right) \geq\|x\|_{\alpha}^{2}-\rho_{0}^{2} .
\end{aligned}
$$

Recalling (21), we infer

$$
\|u(s, x)\|_{\alpha} \leq B e^{-\lambda s}\|x\|_{\alpha}+\rho \leq B\|x\|_{\alpha}, \quad \forall s \geq 0 .
$$

Frequently, by the definition of $V_{2}$ and the monotonicity property of $\gamma(s)$, we get

$$
\begin{aligned}
V_{2}(x) & \leq \gamma\left(B\|x\|_{\alpha}+\rho\right) \\
& \leq\left(B\|x\|_{\alpha}+\rho\right)^{2} \\
& \leq 2 B^{2}\|x\|_{\alpha}^{2}+2 \rho^{2} .
\end{aligned}
$$

So, we verify the correctness of (38).

Lastly we need to check that $V_{2}(u(t, x))$ is nonincreasing in $t$. Note that

$$
\begin{aligned}
V_{2}(u(t, x)) & =\sup _{s \geq 0} \gamma\left(\|u(s, u(t, x))\|_{\alpha}\right) \\
& =\sup _{s \geq 0} \gamma\left(\|u(s+t, x)\|_{\alpha}\right) \\
& =\sup _{s \geq t} \gamma\left(\|u(s, x)\|_{\alpha}\right) .
\end{aligned}
$$

It is easy to see the validity of our checking.

Now, let

$$
V(x)=V_{1}(x)+V_{2}(x) .
$$

Considering (23), (38), (25), (37), and (31), we can get the validity of (18), (19), and (20). The proof is complete.

In order to prove the second result, we need to verify the following lemma.

Lemma 5. Suppose that $f$ is globally Lipschitz with Lipschitz constant $L>0$, that is,

$$
\left\|f\left(x_{1}, x_{2}\right)-f\left(y_{1}, y_{2}\right)\right\| \leq L\left(\left\|x_{1}-y_{1}\right\|_{\alpha}+\left\|x_{2}-y_{2}\right\|_{\alpha}\right),
$$

for any $x_{i}, y_{i} \in X^{\alpha}$, and that the system (2) is exponentially dissipative. Then, there exist $B_{0}>1$ and $\tau_{0}>0$ such that when $\tau \leq \tau_{0}$, any solution of (1) with initial value $u_{0} \in \mathscr{C}=$ $C\left([-\tau, 0], X^{\alpha}\right)$ satisfies

$$
\left\|u\left(t, u_{0}\right)\right\|_{\alpha}<B_{0}\left(\mid\left\|u_{0}\right\|_{\alpha}+1\right), \quad \forall t \geq 0, u_{0} \in \mathscr{C} .
$$

Proof. According to (45), it is easy to see that there is an $M>$ 0 such that

$$
\|f(x, y)\| \leq M\left(\|x\|_{\alpha}+\|y\|_{\alpha}+1\right), \quad \forall x, y \in X^{\alpha} .
$$

Firstly, we prove that for arbitrary $t \in[0, \tau]$, there exists $B_{1}$ such that any solution of (1) with initial value $u_{0} \in \mathscr{C}=$ $C\left([-\tau, 0], X^{\alpha}\right)$ satisfies

$$
\left\|u\left(t, u_{0}\right)\right\|_{\alpha}<B_{1}\left(\mid\left\|u_{0}\right\|_{\alpha}+1\right),
$$

because $u\left(t, u_{0}\right)$ can be expressed as follows:

$$
\begin{aligned}
u(t) & =e^{-t A} u_{0}(0)+\int_{0}^{t} e^{-(t-s) A} f(u(s), u(s-\tau)) d s \\
& =e^{-t A} u_{0}(0)+\int_{0}^{t} e^{-(t-s) A} f\left(u(s), u_{0}(s-\tau)\right) d s
\end{aligned}
$$


By (47) and (15), we can obtain

$$
\begin{aligned}
\|u(t)\|_{\alpha}= & \left\|A^{\alpha} u(t)\right\| \leq\left\|e^{-t A} A^{\alpha} u_{0}(0)\right\|+\int_{0}^{t}\left\|A^{\alpha} e^{-(t-s) A}\right\| \\
& \cdot M\left(\|u(s)\|_{\alpha}+\left\|u_{0}(s-\tau)\right\|_{\alpha}+1\right) d s \\
\leq & C_{1} \mid\left\|u_{0}\right\|_{\alpha}+M C_{\alpha} \int_{0}^{t}(t-s)^{-\alpha}\|u(s)\|_{\alpha} d s \\
& +M C_{\alpha}\left|\left\|u_{0}\right\|\right|_{\alpha} \int_{0}^{t}(t-s)^{-\alpha} d s \\
& +M\left|\left\|u_{0}\right\|\right|_{\alpha} \int_{0}^{t}(t-s)^{-\alpha} d s \\
\leq & C_{2} \mid\left\|u_{0}\right\|\left\|_{\alpha}+M C_{\alpha} \int_{0}^{t}(t-s)^{-\alpha}\right\| u_{0}(s) \|_{\alpha} d s .
\end{aligned}
$$

According to the Gronwall inequality, one easily sees that

$$
\left\|u\left(t, u_{0}\right)\right\|_{\alpha} \leq C_{3}\left|\left\|u_{0}\right\|\right|_{\alpha}<B_{1}\left(\left|\left\|u_{0}\right\|\right|_{\alpha}+1\right) .
$$

Now, we choose and fix $B_{2}$ and $\tau_{0}$ with

$$
B_{2}>\sqrt{\frac{3(b d+a d+c d+b \sigma+c)}{d}}, \quad \tau_{0}^{\delta}<\frac{d}{96 b M_{0} L_{V} L} .
$$

Let $v(t)=u\left(t, u_{0}\right)$. We will show that

$$
\|v(t)\|_{\alpha}<B_{2}\left(\left\|v_{\tau}\right\|_{\alpha}+1\right), \quad \forall t \geq \tau
$$

where $\left\|v_{\tau}\right\|_{\alpha}=\max _{[0, \tau]}\|v(s)\|_{\alpha}$.

We argue by contradiction and suppose that for some solution $v(t)=u\left(t, u_{0}\right)$ of $(1)$, it holds that

$$
\left\|v\left(t_{1}\right)\right\|_{\alpha} \geq B_{2}\left(\left\|v_{\tau}\right\|_{\alpha}+1\right)
$$

for some $t_{1}>\tau$. Observing that $B_{2}>1$, we deduce that there exists a $t_{0}>\tau$ such that

$$
\begin{gathered}
\|v(t)\|_{\alpha}<B_{2}\left(\left\|v_{\tau}\right\|_{\alpha}+1\right), \quad \text { for } t \in\left[\tau, t_{0}\right), \\
\left\|v\left(t_{0}\right)\right\|_{\alpha}=B_{2}\left(\left\|v_{\tau}\right\|_{\alpha}+1\right) .
\end{gathered}
$$

Thanks to Theorem 4, there is a Lyapunov function $V$ satisfying (18)-(20). By Lemma 2, we find that

$$
\begin{gathered}
\frac{d^{+}}{d t} V(v(t)) \\
=\limsup _{h \rightarrow 0+} \frac{1}{h}[V(v(t)+h f(v(t), v(t-\tau))-A v(t)) \\
-V(v(t))]
\end{gathered}
$$

$$
\begin{gathered}
\leq \limsup _{h \rightarrow 0+} \frac{1}{h}[V(v(t)+h F(v(t))-A v(t))-V(v(t))] \\
+\limsup _{h \rightarrow 0+} \frac{1}{h}[V(v(t)+h f(v(t), v(t-\tau))) \\
-V(v(t)+h F(v(t))] \\
=D_{g(v(t))}^{+} V(v(t)) \\
+\limsup _{h \rightarrow 0+} \frac{1}{h}[V(v(t)+h f(v(t), v(t-\tau))) \\
-V(v(t)+h F(v(t))] .
\end{gathered}
$$

By (47) and (55), we see that for $t \in\left[\tau, t_{0}\right]$

$$
\begin{aligned}
& \|F(v(t))\|,\|f(v(t), v(t-\tau))\| \\
& \quad \leq M\left[2 B_{2}\left(\left\|v_{\tau}\right\|_{\alpha}+1\right)+1\right]=R .
\end{aligned}
$$

Denote by $L_{h}$ the Lipschitz constant of $V$ on $\overline{\mathscr{B}}(v(t), h R)$. Then, we infer from (20) that

$$
\limsup _{h \rightarrow 0+} L_{h} \leq L_{V}\left(2\|v(t)\|_{\alpha}+1\right) .
$$

At the same time, from Lemma 3.3.2 of [18], we can show that $v(t)$ is locally Hölder. That is to say,

$$
\|v(t-\tau)-v(t)\|_{\alpha} \leq M_{0} \tau^{\delta}, \quad \delta \in(0,1-\alpha) .
$$

Therefore, on $\left[\tau, t_{0}\right]$ we have that

$$
\begin{aligned}
\frac{d^{+}}{d t} V(v(t)) & \\
\leq & D_{g(v(t))}^{+} V(v(t)) \\
& \quad+\limsup _{h \rightarrow 0+} L_{h}\|f(v(t), v(t-\tau))-f(v(t), v(t))\| \\
\leq & D_{g(v(t))}^{+} V(v(t))+\limsup _{h \rightarrow 0+} L\|v(t-\tau)-v(t)\|_{\alpha} \\
\leq & -d\|v(t)\|_{\alpha}^{2}+\sigma+M_{0} \tau^{\delta} L L_{V}\left(2\|v(t)\|_{\alpha}+1\right) \\
\leq & -d\|v(t)\|_{\alpha}^{2}+\sigma+M_{0} \tau^{\delta} L L_{V}\left[2 B_{2}\left(\left\|v_{\tau}\right\|_{\alpha}+1\right)+1\right]
\end{aligned}
$$

because

$$
\begin{aligned}
{\left[2 B_{2}\right.} & \left.\left(\left\|v_{\tau}\right\|_{\alpha}+1\right)+1\right] \\
& \leq\left[2 B_{2}\left(\left\|v_{\tau}\right\|_{\alpha}+1\right)+1\right]^{2} \\
& \leq\left[2 B_{2}\left(\left\|v_{\tau}\right\|_{\alpha}+1\right)+2 B_{2}\right]^{2} \\
& =4 B_{2}^{2}\left(\left\|v_{\tau}\right\|_{\alpha}+2\right)^{2} \\
& \leq 16 B_{2}^{2}\left(\left\|v_{\tau}\right\|_{\alpha}+1\right)^{2} .
\end{aligned}
$$


According to (18) and (61), we find that

$$
\begin{aligned}
\frac{d^{+}}{d t} V(v(t)) \leq & -\frac{d}{b} V(v(t))+\frac{d c}{b}+\sigma \\
& +32 M_{0} \tau^{\delta} L L_{V} B_{2}^{2}\left(\left\|v_{\tau}\right\|_{\alpha}+1\right)^{2}, \quad \forall t \in\left[\tau, t_{0}\right] .
\end{aligned}
$$

If we denote that

$$
\begin{gathered}
\lambda_{1}=\frac{d}{b}, \quad \sigma_{1}=\frac{c d}{b}, \\
C\left(\left\|v_{\tau}\right\|_{\alpha}\right)=32 M_{0} \tau^{\delta} L L_{V} B_{2}^{2}\left(\left\|v_{\tau}\right\|_{\alpha}+1\right)^{2},
\end{gathered}
$$

then by the Gronwall inequality

$$
\begin{aligned}
V(v(t)) \leq & V(v(\tau)) e^{-\lambda_{1}(t-\tau)} \\
& \quad+\frac{1}{\lambda_{1}}\left[\sigma_{1}+C\left(\left\|v_{\tau}\right\|_{\alpha}\right)\right]\left(1-e^{-\lambda_{1}(t-\tau)}\right) \\
\leq & V(v(\tau))+\frac{1}{\lambda_{1}}\left[\sigma_{1}+C\left(\left\|v_{\tau}\right\|_{\alpha}\right)\right] .
\end{aligned}
$$

Utilizing (18) again, we conclude that for $t \in\left[\tau, t_{0}\right]$,

$$
\begin{aligned}
\|v(t)\|_{\alpha}^{2} \leq & b\left\|v_{\tau}\right\|_{\alpha}^{2}+\left(a+c+\frac{\sigma_{1}}{\lambda_{1}}\right) \\
& +\frac{32}{\lambda_{1}} M_{0} \tau^{\delta} L L_{V} B_{2}^{2}\left(\left\|v_{\tau}\right\|_{\alpha}+1\right)^{2} .
\end{aligned}
$$

By the choice of $B_{1}$ and $\tau_{0}$, one easily checks that

$$
\begin{gathered}
b<\frac{1}{3} B_{2}^{2}, \quad a+c+\frac{\sigma_{1}}{\lambda_{1}}<\frac{1}{3} B_{2}^{2}, \\
\frac{32}{\lambda_{1}} M_{0} \tau^{\delta} L L_{V}<\frac{1}{3} .
\end{gathered}
$$

Hence, in particular, for $t=t_{0}$, we find that

$$
\left\|v\left(t_{0}\right)\right\|_{\alpha}^{2}<B_{2}^{2}\left(\left\|v_{\tau}\right\|_{\alpha}+1\right)^{2} .
$$

This contradicts (56).

Now, the conclusion of the theorem follows immediately from (48) and (53). And the proof is complete.

Theorem 6. Assume that $f$ is globally Lipschitz and the system (2) without delay is exponentially dissipative. Then, the system (1) with time delay is also exponentially dissipative.

Proof. Let $u_{0} \in \mathscr{C}$ and $v(t)=u\left(t, u_{0}\right)$ be the solution of (1). According to Lemma 5, $\|v(t)\|_{\alpha} \leq B_{1}\left(\left\|v_{\tau}\right\|_{\alpha}+1\right)$ for all $t \geq \tau$, repeating the same argument as in (65), one easily sees that the first inequality in (65) remains valid for all $t \geq \tau$. Furthermore, making use of (18), we deduce that

$$
\begin{aligned}
\|v(t)\|_{\alpha}^{2}-a & \leq V(v(t)) \\
& \leq V(v(\tau)) e^{-\lambda_{1}(t-\tau)}+\frac{1}{\lambda_{1}}\left[\sigma_{1}+C\left(\left\|v_{\tau}\right\|_{\alpha}\right)\right] \\
& \leq\left(b\left\|v_{\tau}\right\|_{\alpha}^{2}+c\right) e^{-\lambda_{1}(t-\tau)}+\frac{1}{\lambda_{1}}\left[\sigma_{1}+C\left(\left\|v_{\tau}\right\|_{\alpha}\right)\right] .
\end{aligned}
$$

Frequently,

$$
\|v(t)\|_{\alpha}^{2} \leq\left(b\left\|v_{\tau}\right\|_{\alpha}^{2}+c\right) e^{-\lambda_{1}(t-\tau)}+a+\frac{1}{\lambda_{1}}\left[\sigma_{1}+C\left(\left\|v_{\tau}\right\|_{\alpha}\right)\right],
$$

By (46), it can be easily seen that

$$
\begin{aligned}
\|v(t)\|_{\alpha}^{2} & \\
\leq & C_{1}\left(\left|\left\|u_{0}\right\|\right|_{\alpha}+1\right)^{2} e^{-\lambda_{1}}(t-\tau) \\
& +\tau^{\delta} C_{2}\left(\mid\left\|u_{0}\right\|_{\alpha}+1\right)^{2}+C_{3} \\
\leq & C_{1} e^{\lambda_{1} \tau_{0}}\left(\left|\left\|u_{0}\right\|\right|_{\alpha}+1\right)^{2} e^{-\lambda_{1} t} \\
& +\tau^{\delta} C_{2}\left(\left|\left\|u_{0}\right\|\right|_{\alpha}+1\right)^{2}+C_{3}, \quad \forall t \geq \tau,
\end{aligned}
$$

where $C_{i}(i=1,2,3)$ are appropriate positive constants independent of $\tau$ and $u_{0}$. For $t \in[0, \tau]$, we have by (46) that

$$
\|v(t)\|_{\alpha}^{2} \leq B_{0}^{2} e^{\lambda_{1} \tau_{0}}\left(\mid\left\|u_{0}\right\| \|_{\alpha}+1\right)^{2} e^{-\lambda_{1} t}
$$

Therefore, taking $C_{1}^{\prime}=\left(C_{1}+B_{0}^{2}\right) e^{\lambda_{1} \tau_{0}}$, one concludes that

$$
\begin{aligned}
\|v(t)\|_{\alpha}^{2} \leq & C_{1}^{\prime}\left(\left|\left\|u_{0}\right\|\right|_{\alpha}+1\right)^{2} e^{-\lambda_{1} t} \\
& +\tau^{\delta} C_{2}\left(\left|\left\|u_{0}\right\|\right|_{\alpha}+1\right)^{2}+C_{3}, \quad \forall t \geq 0 .
\end{aligned}
$$

Now, we fix a $T>0$ and $\tau \leq \tau_{0}$ independent of $u_{0}$ such that

$$
C_{1}^{\prime} e^{-\lambda_{1} T}<\frac{1}{8}, \quad \tau^{\delta} C_{2}<\frac{1}{8}
$$

So,

$$
\begin{aligned}
\|v(t)\|_{\alpha}^{2} & \leq \frac{1}{4}\left(\mid\left\|u_{0}\right\|_{\alpha}+1\right)^{2}+C_{3} \\
& \leq\left(\frac{\left|\left\|u_{0}\right\|\right|_{\alpha}}{2}+\frac{1}{2}+\sqrt{C_{3}}\right)^{2}, \quad \forall t \geq T .
\end{aligned}
$$

Setting $C_{0}=1 / 2+\sqrt{C_{3}}$, we find that

$$
\|v(t)\|_{\alpha} \leq \frac{1}{2}\left|\left\|u_{0}\right\|\right|_{\alpha}+C_{0}, \quad \forall t \geq T, u_{0} \in \mathscr{C} .
$$

Next, we will use mathematical induction to prove that

$$
\begin{aligned}
\|v(t)\|_{\alpha} \leq & \frac{1}{2^{k}}\left|\left\|u_{0}\right\|\right|_{\alpha} \\
& +C_{0}\left(1+\frac{1}{2}+\cdots+\frac{1}{2^{k-1}}\right), \quad \forall t \geq T_{k}, u_{0} \in \mathscr{C},
\end{aligned}
$$

where $T_{k}=k(T+1)$. 
Indeed, let $u_{0} \in \mathscr{C}$. If $k=1$, then (77) clearly holds true. Suppose that (77) holds for $k=m$; that is,

$$
\begin{aligned}
\|v(t)\|_{\alpha} \leq & \frac{1}{2^{m}} \mid\left\|u_{0}\right\|_{\alpha} \\
& +C_{0}\left(1+\frac{1}{2}+\cdots+\frac{1}{2^{m-1}}\right), \quad \forall t \geq T_{m}, u_{0} \in \mathscr{C} .
\end{aligned}
$$

Then, in particular,

$$
\begin{aligned}
\|v(t)\|_{\alpha} \leq & \frac{1}{2^{m}} \mid\left\|u_{0}\right\|_{\alpha} \\
& +C_{0}\left(1+\frac{1}{2}+\cdots+\frac{1}{2^{m-1}}\right), \quad \forall t \in\left[T_{m}, T_{m}+\tau\right] .
\end{aligned}
$$

From (76), we know that

$$
\begin{aligned}
& \left\|v\left(t+T_{m}+\tau\right)\right\|_{\alpha} \\
& \quad \leq \frac{1}{2} \max _{t \in[-\tau, 0]}\left\|v\left(t+T_{m}+\tau\right)\right\|_{\alpha}+C_{0}, \quad \forall t \geq T .
\end{aligned}
$$

If we consider $t+T_{m}+\tau$ as $t$, then the above can be rewritten as

$$
\|v(t)\|_{\alpha} \leq \frac{1}{2} \max _{t \in\left[T_{m}, T_{m}+\tau\right]}\|v(t)\|_{\alpha}+C_{0}, \quad \forall t \geq T_{m}+\tau+T .
$$

From (79),

$$
\begin{gathered}
\|v(t)\|_{\alpha} \\
\leq \frac{1}{2}\left[\frac{1}{2^{m}}\left|\left\|u_{0}\right\|\right|_{\alpha}+C_{0}\left(1+\frac{1}{2}+\cdots+\frac{1}{2^{m}-1}\right)\right]+\rho_{0} \\
=\frac{1}{2^{m+1}}\left|\left\|u_{0}\right\|\right|_{\alpha}+C_{0}\left(1+\frac{1}{2}+\cdots+\frac{1}{2^{m}}\right), \\
\forall t \geq T_{m}+\tau+T .
\end{gathered}
$$

Choosing $\tau \leq 1$, we conclude that

$$
\begin{array}{r}
\|v(t)\|_{\alpha} \leq \frac{1}{2^{m+1}} \mid\left\|u_{0}\right\|_{\alpha}+C_{0}\left(1+\frac{1}{2}+\cdots+\frac{1}{2^{m}}\right), \\
\forall t \geq T_{m}+1+T=T_{m+1} .
\end{array}
$$

Thus, we see that (77) holds for $m+1$.

By (77), we know that

$$
\begin{gathered}
\|v(t)\|_{\alpha} \leq \frac{1}{2^{k}} \mid\left\|u_{0}\right\|_{\alpha}+2 C_{0}, \\
\forall t \in\left[T_{k}, T_{k+1}\right], u_{0} \in \mathscr{C}, k=1,2, \ldots .
\end{gathered}
$$

Furthermore, by Lemma 5, we see that

$$
\begin{gathered}
\|v(t)\|_{\alpha} \leq \frac{B_{0}}{2^{k}} \mid\left\|u_{0}\right\|_{\alpha}+\rho_{0}, \\
\forall t \in\left[T_{k}, T_{k+1}\right], u_{0} \in \mathscr{C}, k=0,1,2, \ldots,
\end{gathered}
$$

where $\rho_{0}=2 C_{0}+1$.
We observe that $t /(T+1) \in[k, k+1]$ when $t \in\left[T_{k}, T_{k+1}\right]$, so we infer from (85) that

$$
\begin{aligned}
\|v(t)\|_{\alpha} & \leq \frac{B_{0}}{2^{k}}\left|\left\|u_{0}\right\|\right|_{\alpha}+\rho_{0} \\
& =\frac{2 B_{0}}{2^{k+1}}\left|\left\|u_{0}\right\|\right|_{\alpha}+\rho_{0} \\
& \leq 2 B_{0} 2^{-\alpha t}\left|\left\|u_{0}\right\|\right|_{\alpha}+\rho_{0}, \\
& \forall t \in\left[T_{k}, T_{k+1}\right], u_{0} \in \mathscr{C}, k=0,1,2, \ldots,
\end{aligned}
$$

where $\alpha=1 /(T+1)$. So, we easily see that

$$
\|v(t)\|_{\alpha} \leq 2 B_{0} 2^{-\alpha t}\left|\left\|u_{0}\right\|\right|_{\alpha}+\rho_{0}, \quad \forall t \geq 0, u_{0} \in \mathscr{C} .
$$

This completes the proof of the theorem.

\section{References}

[1] F. Guo, W. Yu, and F. Huang, "Robustness with respect to small delays for exponential stability of non-autonomous systems," Journal of Mathematical Analysis and Applications, vol. 288, no. 2, pp. 671-679, 2003.

[2] O. Arino and M. Pituk, "More on linear differential systems with small delays," Journal of Differential Equations, vol. 170, no. 2, pp. 381-407, 2001.

[3] R. D. Driver, "Linear differential systems with small delays," Journal of Differential Equations, vol. 21, no. 1, pp. 148-166, 1976.

[4] J. K. Hale and S. M. Verduyn Lunel, "Effects of small delays on stability and control," in Operator Theory and Analysis (Amsterdam, 1997), vol. 122 of Oper. Theory Adv. Appl., pp. 275301, Birkhäuser, Basel, Switzerland, 2001.

[5] B. Xu, "Stability robustness bounds for linear systems with multiple time-varying delayed perturbations," International Journal of Systems Science, vol. 28, no. 12, pp. 1311-1317, 1997.

[6] D. Li and Y. Wang, "Asymptotic behavior of gradient systems with small time delays," Nonlinear Analysis: Real World Applications, vol. 11, no. 3, pp. 1627-1633, 2010.

[7] D. Li, "Morse decompositions for general dynamical systems and differential inclusions with applications to control systems," SIAM Journal on Control and Optimization, vol. 46, no. 1, pp. 35-60, 2007.

[8] D. Li and P. E. Kloeden, "Robustness of asymptotic stability to small time delays," Discrete and Continuous Dynamical Systems. Series A, vol. 13, no. 4, pp. 1007-1034, 2005.

[9] G. Friesecke, "Convergence to equilibrium for delay-diffusion equations with small delay," Journal of Dynamics and Differential Equations, vol. 5, no. 1, pp. 89-103, 1993.

[10] X. Mao, "Exponential stability of nonlinear differential delay equations," Systems \& Control Letters, vol. 28, no. 3, pp. 159-165, 1996.

[11] A. M. Lyapunov, The General Problem of the Stability of Motion, Kharkov Mathematical Society, Lyapunov, Russia, 1892.

[12] T. Yoshizawa, Stability Theory by Lyapunov's Second Method, Mathematical Society of Japan, 1996.

[13] Y. Guo and X. Li, "A converse lyapunov theorem and robustness with respect to unbounded perturbations for exponential dissipativity," Advances in Difference Equations, vol. 2010, Article ID 726347, 15 pages, 2010. 
[14] J. K. Hale, Asymptotic Behavior of Dissipative Systems, vol. 25 of Mathematical Surveys and Monographs, American Mathematical Society, Providence, RI, USA, 1988.

[15] J. C. Robinson, Infinite-Dimensional Dynamical Systems, Cambridge Texts in Applied Mathematics, Cambridge University Press, Cambridge, UK, 2001.

[16] X. X. Liao, Stability of Theories, Methods and Applications, Hua Zhong University Science and Technology Press, 1998.

[17] G. R. Sell and Y. You, Dynamics of Evolutionary Equations, vol. 143 of Applied Mathematical Sciences, Springer, New York, NY, USA, 2002.

[18] D. Henry, Geometric Theory of Semilinear Parabolic Equations, vol. 840 of Lecture Notes in Mathematics, Springer, Berlin, Germany, 1981. 


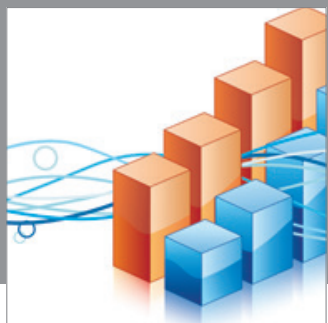

Advances in

Operations Research

mansans

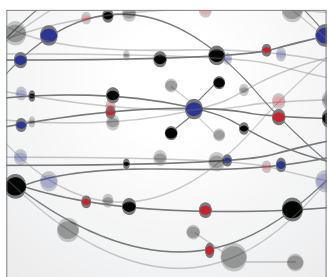

The Scientific World Journal
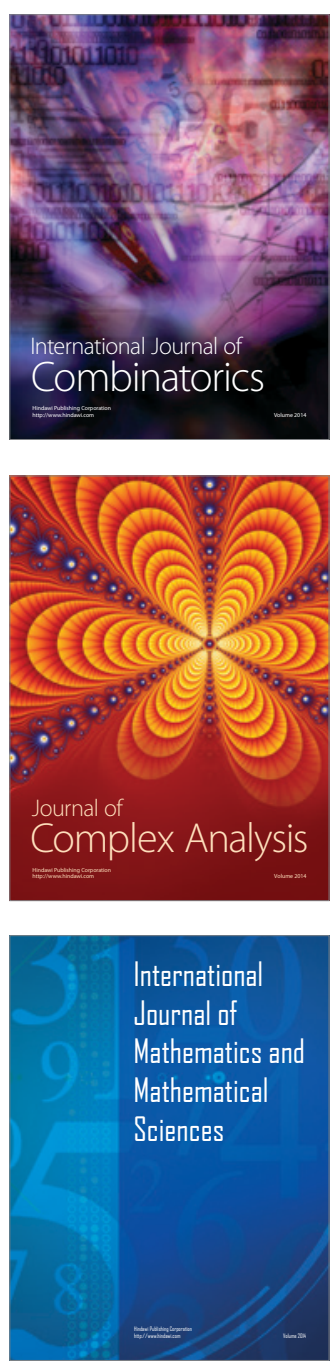
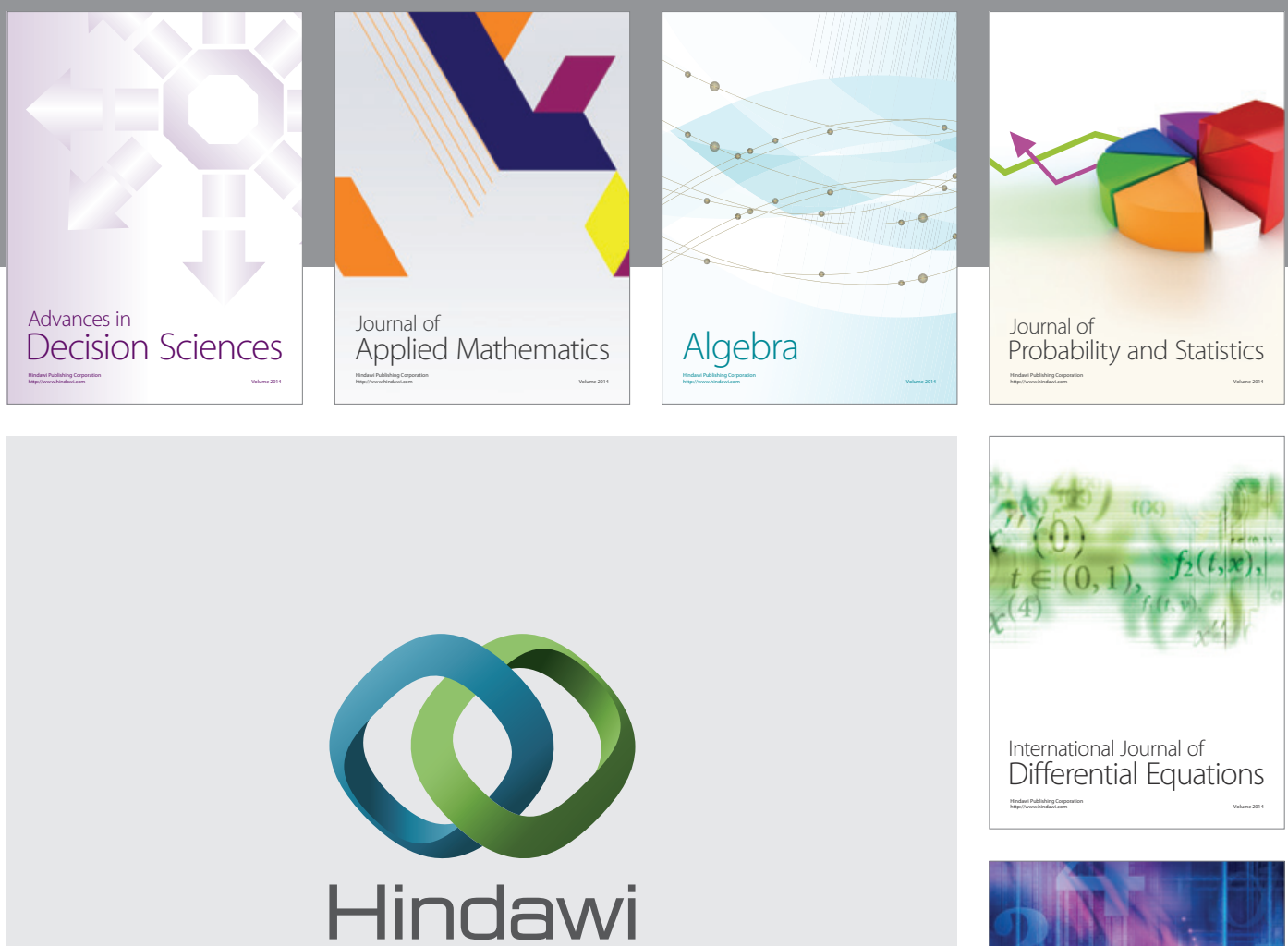

Submit your manuscripts at http://www.hindawi.com
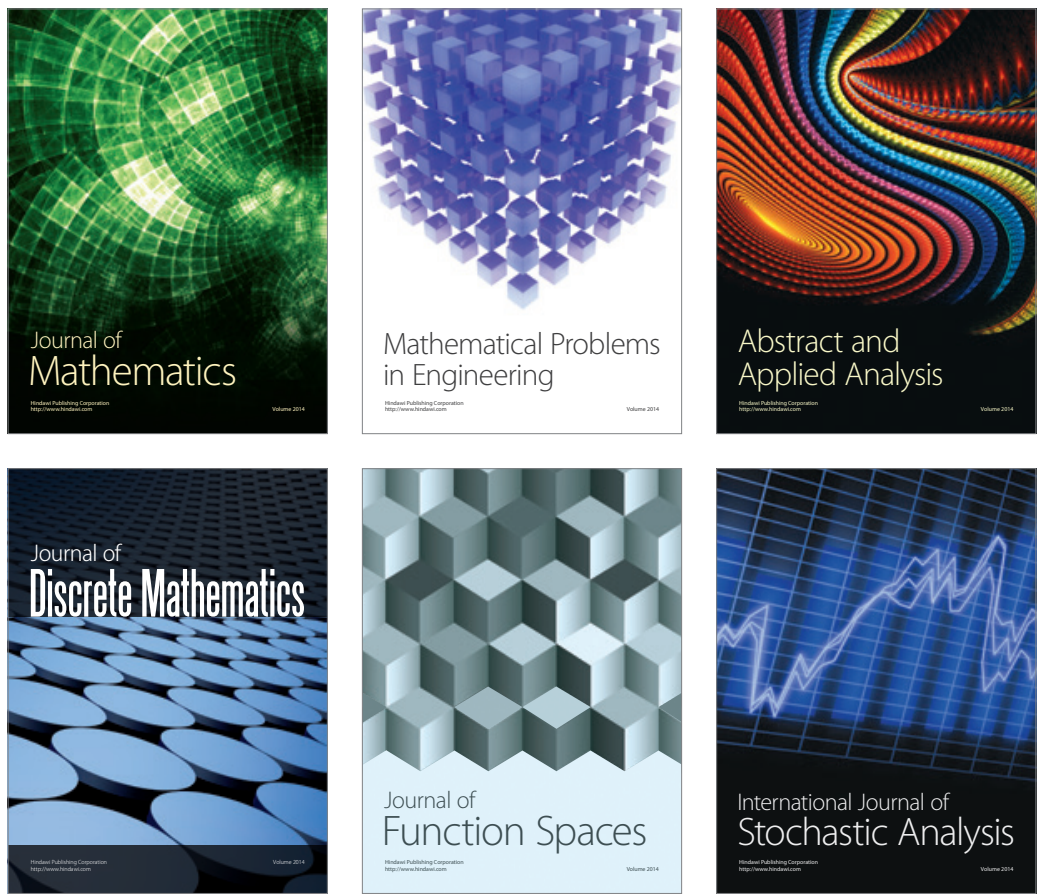

Journal of

Function Spaces

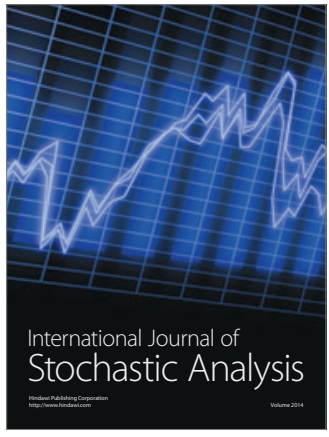

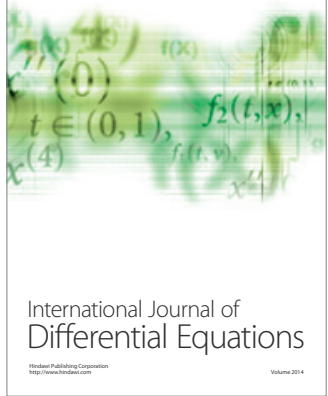
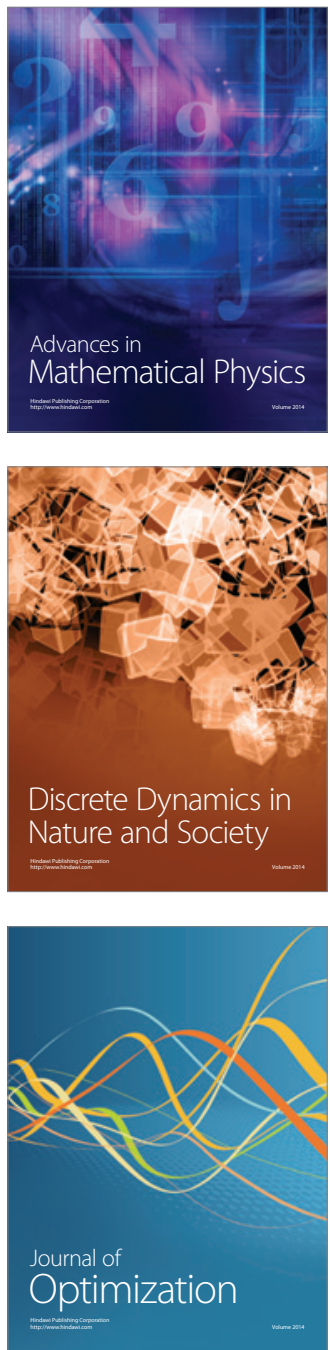